\title{
Restauração Florestal de uma Mina de Bauxita: Avaliação do Desenvolvimento das Espécies Arbóreas Plantadas
}

\author{
Kelly de Almeida Silva ${ }^{1 \star}$, Sebastião Venâncio Martins ${ }^{1}$, Aurino Miranda Neto ${ }^{1}$, \\ Raul de Abreu Demolinari², Aldo Teixeira Lopes ${ }^{2}$ \\ ${ }^{1}$ Departamento de Engenharia Florestal, Universidade Federal de Viçosa - UFV, Viçosa/MG, Brasil \\ ${ }^{2}$ Votorantim Metais, Miraí/MG, Brasil
}

\begin{abstract}
RESUMO
O objetivo deste estudo foi avaliar as mudas de espécies arbóreas plantadas para fins de restauração florestal em uma área pós-mineração de bauxita. Foram alocadas 20 parcelas de $9 \times 6 \mathrm{~m}$ nas quais foram mensurados diâmetro ao nível do solo, altura e diâmetro da copa das mudas plantadas. Também foram calculados a porcentagem de mortalidade e o valor de importância (VI) das espécies. Foram registrados 540 indivíduos arbóreos vivos (22,9\% de mortalidade) e 45 espécies. As espécies com maiores VIs foram Solanum lycocarpum (14,7\%) e Schinus terebinthifolius (10,8\%). S. terebinthifolius, S. lycocarpum e Joannesia princeps contribuíram com $30,4 \%$ de cobertura de copa. A altura média das mudas plantadas variou de $0,40 \mathrm{~m}$ a 3,90 $\mathrm{m}$. As espécies utilizadas na restauração da área minerada proporcionaram benefícios ecológicos para a área, como cobertura do solo, atenuando processos erosivos e a invasão por gramíneas exóticas agressivas.
\end{abstract}

Palavras-chave: área degradada, mineração, restauração ecológica.

\section{Forest Restoration After Bauxite Mining: Assessment of Planted tree Species}

\begin{abstract}
The aim of this study was to evaluate the seedlings of tree species planted for forest restoration in a bauxite mining. We allocated twenty plots of $9 \times 6 \mathrm{~m}$ and diameter at ground level, height and canopy diameter of planted seedlings. Also were calculated the mortality percentage and the importance value (VI) species. We recorded 540 alive individuals $(22.86 \%$ mortality) and 45 species. The species with the highest VIs were Solanum lycocarpum (14.7\%) and Schinus terebinthifolius (10.8\%). S. terebinthifolius, S. lycocarpum and Joannesia princeps, contributed $30.4 \%$ canopy cover. Average height of seedlings planted ranged from $0.4 \mathrm{~m}$ to $3.9 \mathrm{~m}$. The species used in the restoration of the mined area provided ecological benefits to the area, such as soil coverage, thus ameliorating erosion and invasion of aggressive exotic grasses.
\end{abstract}

Keywords: degraded area, mining, ecological restoration. 


\section{INTRODUÇÃO}

Os depósitos de bauxita no território brasileiro correspondem a cerca de $10 \%$ das reservas mundiais, com 2,6 bilhões de toneladas, ocupando o país o quarto lugar entre os países produtores (USGS, 2015). A exploração mineral de bauxita demanda a supressão da vegetação e a remoção dos horizontes superficiais do solo (Grant et al., 2007), sendo necessária a adoção de medidas mitigadoras e compensatórias.

Nesse cenário, além da compensação ambiental em áreas próximas do local minerado, torna-se necessária a restauração do ecossistema degradado. A restauração florestal visa a criação de comunidades sustentáveis e representativas da composição e da diversidade das formações florestais em que a área degradada se insere (Jefferson, 2004; Courtney et al., 2009). Essa medida promove o avanço temporal dos processos ecológicos, da estrutura e da dinâmica sucessional, no sentido de que o novo ecossistema se aproxime em termos ecológicos do ecossistema de referência (Chazdon, 2008; Martins, 2014).

A avaliação e o monitoramento de áreas em processo de restauração são importantes para verificar o seu desenvolvimento, a identificação de perturbações e a consequente necessidade de interferência por meio de manejo ou replantios. Monitoramento e avaliação são também necessários para a avaliação da eficiência dos modelos e espécies empregadas e para subsidiar futuros estudos de restauração ecológica (Ignácio et al., 2007; Rodrigues et al., 2009).

Entre os parâmetros analisados em relação aos indivíduos plantados avaliam-se: aspecto visual, densidade de plantas, altura média de plantas (Almeida \& Sánchez, 2005), composição de espécies, cobertura de copa, classificação das espécies em grupos sucessionais, origem (espécies nativas, exóticas, invasoras), síndromes de dispersão e taxa de mortalidade (Brancalion et al., 2015).

A análise florística e estrutural do estrato arbóreo de ecossistemas em processo de restauração permite um melhor conhecimento do comportamento dessas espécies e propicia um melhor subsídio para inferir se esses ecossistemas apresentam respostas positivas quanto ao processo de sucessão que se deseja acelerar (Miranda et al., 2012).
Assim, o objetivo deste estudo foi verificar a situação das espécies arbóreas de um projeto de restauração florestal em área de mineração de bauxita após 18 meses do plantio.

\section{MATERIAL E MÉTODOS}

\section{1. Área de estudo}

O estudo foi realizado em uma área em processo de restauração por meio de plantio de mudas em área total, localizada no município de Descoberto, sudeste de Minas Gerais ( $21^{\circ} 25^{\prime} 42^{\prime \prime} \mathrm{S}$ e $\left.42^{\circ} 56^{\prime} 07^{\prime \prime} \mathrm{W}\right)$, com altitude entre 670 e $715 \mathrm{~m}$. A área de estudo possui 4 ha e estava em processo de restauração há 18 meses no momento do início dos levantamentos. Em seu entorno, há áreas restauradas com diferentes idade, pastagens e fragmentos florestais preservados.

O clima da região, segundo a classificação de Köppen, é do tipo Aw, tropical com estação seca no inverno (Sá, 2009). A temperatura é alta no verão, atingindo os $40{ }^{\circ} \mathrm{C}$, diminuindo para $20-22{ }^{\circ} \mathrm{C}$ no inverno, e a precipitação pluviométrica média anual é de $1.300 \mathrm{~mm}$ (Lopes \& Branquinho, 1988).

O relevo da região é bastante acidentado, com pequenas planícies e platôs semidissecados, limitados por franjas escarpadas e montanhosas, com vales retilíneos (Lopes \& Branquinho, 1988). A vegetação característica da região é classificada como Floresta Estacional Semidecidual Montana, inserida no domínio Mata Atlântica (IBGE, 2012).

Posteriormente à mineração, teve início a recuperação da área no ano de 2011, seguindo as etapas: recomposição topográfica em curvas de nível, deposição da camada fértil de solo (topsoil de 0,3 m), retirada anteriormente à mineração, subsolagem nas linhas de plantio, calagem para correção da acidez do solo, adubação fosfatada e adubação de base. Posteriormente foram semeados $200 \mathrm{~kg} \mathrm{ha}^{-1}$ da leguminosa Cajanus cajan (feijão guandu), com a função de fixar biologicamente nitrogênio e adubo verde, realizada adubação de cobertura, no entorno de cada muda, e combate periódico a formigas, com isca granulada.

O plantio das mudas de espécies arbóreas foi realizado no espaçamento de $1,5 \times 1,5 \mathrm{~m}$ (4.444 indivíduos ha-1). Esse espaçamento adensado foi indicado para promover a rápida cobertura do topsoil depositado, que estava sujeito a processos erosivos. 


\subsection{Avaliação inicial do reflorestamento}

A avaliação do plantio foi realizada 18 meses após o plantio das mudas. As espécies foram classificadas em famílias e tiveram os nomes científicos atualizados de acordo com o sistema do Angiosperm Phylogeny Group III (APG, 2009) e pela base de dados da Lista de Espécies da Flora do Brasil (JBRJ, 2014). Parâmetros fitossociológicos como densidade relativa, dominância relativa, frequência relativa e valor de importância (Mueller-Dombois \& Ellenberg, 1974) para descrever a estrutura da comunidade foram calculados por meio do programa FITOPAC 2.1 (Shepherd, 2010).

Para a avaliação das características estruturais dos indivíduos plantados foram alocadas 20 parcelas de $9 \times 6 \mathrm{~m}$, com distância de $10 \mathrm{~m}$ entre parcelas, conforme metodologia proposta por Brancalion et al. (2015). Das mudas plantadas presentes na área amostral foram mensurados o diâmetro no nível do solo (DNS), a altura e o diâmetro de copa.

A taxa de mortalidade foi obtida de acordo com o proposto por Brancalion et al. (2015), através da proporção de plantas mortas presentes na unidade amostral, sendo consideradas plantas mortas aquelas ausentes do local determinado da cova ou pelas plantas que apresentaram o caule seco e desprovido de folhas.

A avaliação da cobertura de copa foi feita pelo método de mensuração da projeção da área da copa, determinada pela área da projeção vertical da copa de cada indivíduo arbóreo, por meio de seu diâmetro com auxílio de uma trena, de acordo com a Equação 1 (Moro \& Martins, 2011).

$C C e=100(\mathrm{Ce} / \mathrm{A})$

em que: $C C e=$ cobertura de copas de dada espécie $e$; $\mathrm{Ce}=$ soma das áreas ocupadas pela projeção das copas de todos os indivíduos da espécie $e, \mathrm{em} \mathrm{m}^{2} ; A$ = área total amostrada, em $\mathrm{m}^{2}$.

Foi obtida também a cobertura de copas total da comunidade, de acordo com a Equação 2 (Moro \& Martins, 2011).

$C C T=100(C i / A)$

em que: $C C T=$ cobertura de copas total da comunidade; $C i=$ soma das áreas ocupadas pela projeção das copas de todos os indivíduos amostrados, $\mathrm{em} \mathrm{m}^{2} ; A$ = área total amostrada, em $\mathrm{m}^{2}$.

\subsection{Categoria sucessional, sindrome de dispersão e ocorrência das espécies}

As espécies plantadas foram classificadas quanto ao grupo sucessional, de acordo com o proposto por Gandolfi et al. (1995), em pioneiras, secundárias iniciais, secundárias tardias e não classificadas, com o objetivo de aumentar o nível de informações sobre a autoecologia das espécies e auxiliar na discussão sobre a dinâmica da sucessão da vegetação estudada. As espécies foram também classificadas quanto à síndrome de dispersão de sementes em zoocóricas, anemocóricas, autocóricas (Pijl, 1982) e quanto à sua ocorrência em nativas do Brasil, nativas regionais e exóticas (conforme Martins \& Kunz, 2007).

\section{RESULTADOS E DISCUSSÃO}

\subsection{Avaliação das espécies do plantio}

Foram registrados na área amostral 540 indivíduos arbóreos vivos, dos 700 plantados, pertencentes a 45 espécies e 18 famílias (Tabela 1). Portanto, houve uma porcentagem de mortalidade de $22,9 \%$, valor que pode ser considerado baixo quando comparado com outras avaliações realizadas em projetos de restauração da Mata Atlântica.

Em uma área em processo de restauração por meio de plantio no espaçamento $3 \times 4 \mathrm{~m}$ localizado na região do Pontal do Paranapanema, SP, com histórico de uso em monocultivo e pastagens, foram encontrados na amostragem 868 indivíduos arbóreos vivos, distribuídos em 38 espécies e 20 famílias, e uma percentagem de mortalidade de 50,2\% também após 18 meses de implantação da restauração (Rodrigues et al., 2010).

O presente estudo e o estudo citado acima, estão situados em região de Mata Atlântica com vegetação característica de Floresta Estacional Semidecidual, porém o clima da área de estudo de Rodrigues et al. (2010) é, segundo classificação de Köppen, do tipo Cwa, mas também de inverno seco.

Em uma área restaurada por meio do plantio de mudas nativas no espaçamento $2 \times 3 \mathrm{~m}$ em que o uso anterior era pastagem, em Sorocaba, SP, e na qual foram plantados 1.413 indivíduos ha-1 ${ }^{-1}$ distribuídos em 59 espécies e 26 famílias, a avaliação da área após 2 anos de restauração constatou um percentual de mortalidade de $31,3 \%$ dos indivíduos plantados (Schievenin et al., 2012). 
Tabela 1. Relação das espécies do plantio registradas na área em restauração.

Table 1. List of species of planting registered in the restoration site.

\begin{tabular}{|c|c|c|c|c|c|}
\hline Família & Espécies plantadas & NI & CS & SD & Oc \\
\hline Anacardiaceae & Schinus terebinthifolius Raddi & 82 & $\mathrm{P}$ & Zoo & NR \\
\hline Annonaceae & Annona muricata $\mathrm{L}$. & 1 & NC & NC & $\mathrm{E}$ \\
\hline \multirow{2}{*}{ Bignoniaceae } & Cybistax antisyphilitica (Mart.) Mart. & 1 & $\mathrm{Si}$ & Ane & NR \\
\hline & Zeyheria tuberculosa (Vell.) Bureau ex. Verl. & 3 & $\mathrm{Si}$ & Ane & NR \\
\hline Boraginaceae & Cordia magnoliifolia Cham. & 1 & $\mathrm{Si}$ & NC & $\mathrm{NB}$ \\
\hline Combretaceae & Terminalia catappa L. & 14 & NC & Zoo & $\mathrm{E}$ \\
\hline \multirow{2}{*}{ Euphorbiaceae } & Joannesia princeps Vell. & 40 & $\mathrm{Si}$ & Auto & $\mathrm{NB}$ \\
\hline & Maprounea guianensis Aubl. & 1 & $\mathrm{Si}$ & Auto & NR \\
\hline \multirow{19}{*}{ Fabaceae } & Acacia mangium Willd. & 1 & $\mathrm{P}$ & Zoo & $\mathrm{E}$ \\
\hline & Anadenanthera peregrina (L.) Speg. & 12 & $\mathrm{Si}$ & Ane & NR \\
\hline & Bauhinia forficata Link & 13 & $\mathrm{Si}$ & Auto & NR \\
\hline & Caesalpinia peltophoroides Benth. & 4 & $\mathrm{Si}$ & Auto & $\mathrm{NB}$ \\
\hline & Cassia ferruginea (Schrad.) Schrad. ex DC. & 37 & $\mathrm{Si}$ & Auto & NR \\
\hline & Clitoria fairchildiana R. A. Howard & 31 & $\mathrm{Si}$ & $\mathrm{NC}$ & NB \\
\hline & Enterolobium contortisiliquum (Vell.) Morong & 1 & $\mathrm{P}$ & Zoo & NR \\
\hline & Erythrina falcata Benth. & 15 & $\mathrm{P}$ & Auto & NR \\
\hline & Erythrina verna Vell. & 6 & $\mathrm{P}$ & Auto & NR \\
\hline & Hymenaea courbaril L. & 4 & St & Zoo & NR \\
\hline & Inga vera Willd. & 16 & $\mathrm{Si}$ & Zoo & NR \\
\hline & Leucaena leucocephala (Lam.) de Wit & 12 & $\mathrm{P}$ & NC & $\mathrm{E}$ \\
\hline & Myroxylon peruiferum L. f. & 3 & St & Ane & NR \\
\hline & Peltophorum dubium (Spreng.) Taub. & 3 & $\mathrm{Si}$ & Ane & NR \\
\hline & Piptadenea gonoacantha (Mart.) J. F. Macbr & 31 & $\mathrm{Si}$ & Auto & NR \\
\hline & Plathymenia reticulata Benth. & 5 & $\mathrm{Si}$ & Ane & NB \\
\hline & Schizolobium parahyba (Vell.) Blake & 1 & $\mathrm{P}$ & Ane & NB \\
\hline & Senna macranthera (DC. ex Collad.) H. S. Irwin \& Barneby & 1 & $\mathrm{P}$ & Auto & NR \\
\hline & Senna multijuga (Rich.) H. S. Irwin \& Barneby & 1 & $\mathrm{Si}$ & Zoo & NR \\
\hline Lecythidaceae & Cariniana estrellensis (Raddi) Kuntze & 8 & St & Ane & NR \\
\hline Malpighiaceae & Byrsonima sericea DC. & 1 & $\mathrm{Si}$ & Zoo & NR \\
\hline \multirow{2}{*}{ Malvaceae } & Ceiba speciosa (A. St.-Hil.) Ravenna & 4 & St & Ane & NR \\
\hline & Pachira glabra Pasq. & 11 & $\mathrm{Si}$ & NC & $\mathrm{E}$ \\
\hline \multirow{2}{*}{ Meliaceae } & Cedrela fissilis Vell. & 2 & St & Ane & NR \\
\hline & Guarea macrophylla Vahl & 3 & St & Zoo & NR \\
\hline Myrtaceae & Syzygium cumini (L.) Skeels & 14 & $\mathrm{P}$ & Zoo & $\mathrm{E}$ \\
\hline \multirow{2}{*}{ Phytolaccaceae } & Gallesia integrifolia (Spreng.) Harms & 31 & $\mathrm{Si}$ & Zoo & NR \\
\hline & Seguieria langsdorffii Moq. & 4 & $\mathrm{Si}$ & Zoo & NR \\
\hline Rosaceae & Eriobotrya japonica (Thunb.) Lindl. & 6 & St & Zoo & $\mathrm{E}$ \\
\hline \multirow{3}{*}{ Rubiaceae } & Amaioua guianensis Aubl. & 25 & St & Zoo & NR \\
\hline & Genipa americana L. & 2 & St & Zoo & NR \\
\hline & Genipa infudibuliformis Zappi \& Semir & 10 & NC & Zoo & $\mathrm{NB}$ \\
\hline Sapindaceae & Sapindus saponaria L. & 1 & St & Auto & NB \\
\hline \multirow{3}{*}{ Solanaceae } & Solanum lycocarpum A. St.-Hil & 72 & $\mathrm{P}$ & Zoo & $\mathrm{NR}^{*}$ \\
\hline & Solanum mauritianum Scop. & 1 & $\mathrm{P}$ & Zoo & NR \\
\hline & Solanum paniculatum L. & 3 & $\mathrm{P}$ & Zoo & NR \\
\hline Urticaceae & Cecropia hololeuca Miq. & 2 & $\mathrm{P}$ & Zoo & NR \\
\hline Morta & & 160 & & & \\
\hline Total & & 700 & & & \\
\hline
\end{tabular}

Número de indivíduos (NI); Ocorrência $(\mathrm{Oc})$ : $\mathrm{E}$ = exótica, $\mathrm{NR}$ = nativa regional, NB = nativa do Brasil; Categoria Sucessional $(\mathrm{CS})$ : $\mathrm{P}=$ pioneira, $\mathrm{SI}=$ secundária inicial, $\mathrm{ST}=$ secundária tardia, $\mathrm{NC}=$ não classificada; Síndrome de Dispersão $(\mathrm{SD}):$ Zoo $=$ zoocórica, Ane $=$ anemocórica, Auto $=$ autocórica, $\mathrm{NC}=$ não classificada. ${ }^{\star}$ Espécie do Cerrado mas que ocorre em áreas abertas da Mata Atlântica, comuns em pastagens na região do estudo 
A variação nos dados de mortalidade de áreas restauradas por meio de plantio de mudas nos diferentes estudos citados e neste estudo pode estar relacionada a vários fatores, como o histórico de uso da área restaurada, a presença de espécies invasoras, o grau de degradação da área, a presença de pragas, a escolha das espécies para o plantio, a manutenção da área, entre outros.

Schievenin et al. (2012) relacionaram a alta mortalidade dos indivíduos plantados na área restaurada à falta de manutenção, que permitiu a espécies exóticas agressivas como braquiária (Urochloa decumbens (Stapf) R.D. Webster) e mamona (Ricinus communis L.) atuarem como inibidoras das espécies do plantio, além da compactação do solo, que impediu o desenvolvimento das raízes das plantas, e da utilização de espécies pouco adaptadas às condições de clima e solo do local.

A mortalidade verificada na área deste estudo não é maior que a verificada em outros estudos e também está sendo compensada pela alta densidade de plantio. As mudas que morreram não promoveram falhas significativas no reflorestamento, dado o pequeno espaçamento entre elas.

Além disso, a semeadura de Cajanus cajan (feijão guandu) na área parece estar favorecendo o desenvolvimento das mudas de nativas, fornecendo sombra e melhorando o solo. Cabe lembrar que essa espécie, embora exótica, deve desaparecer naturalmente da área por ser uma espécie anual, como já se verifica em campo com a mortalidade da maioria dos indivíduos.

A adubação verde promove a proteção, melhoria e manutenção da qualidade do solo (Leite et al., 2010), aspectos muito importantes em áreas mineradas. Cajanus cajan é muito utilizada para essa finalidade, pois possui um sistema radicular profundo e ramificado (Azevedo et al., 2007), além de promover um rápido sombreamento da área, impedindo o desenvolvimento de gramíneas invasoras, e de propiciar a fixação biológica de nitrogênio. Isso permite maior disponibilidade do nitrogênio para as plantas que não possuem associação com bactérias fixadoras de nitrogênio.

Ao avaliarem a influência da densidade de Cajanus cajan na mortalidade de espécies nativas plantadas para a restauração florestal, Beltrame \& Rodrigues (2008) verificaram que uma maior densidade de Cajanus cajan aparentemente proporciona uma facilitação inicial das espécies do plantio, relação que pode ser alterada com o tempo. Esses autores também concluíram que a sobrevivência e o incremento em área basal de espécies nativas utilizadas em plantios de restauração florestal podem apresentar melhores resultados no consórcio com Cajanus cajan. Entretanto, as interações positivas desse consórcio dependem da densidade de plantio do Cajanus cajan e da duração do período de consorciação.

Houve uma boa riqueza de espécies no plantio dentro da área amostral, contudo a densidade de indivíduos de muitas das espécies foi baixa (menor que cinco), ao passo que de algumas outras, como Solanum lycocarpum e Schinus terebinthifolius, foi alta (maior que 70 indivíduos) (Tabela 1). Essa tendência de concentração dos indivíduos em poucas espécies pode comprometer os processos ecológicos futuros da área em restauração, através da dominância ecológica de determinadas espécies. Mas como ambas são espécies pioneiras, sendo Solanum lycocarpum de maior ocorrência no domínio do Cerrado e em grandes áreas abertas e antropizadas da Mata Atlântica, é provável que tenham suas populações controladas com o tempo.

Solanum lycocarpum cresce e se desenvolve em condições ambientais desfavoráveis e suporta períodos de seca prolongados (Oliveira-Júnior et al., 2003). Essa espécie é bem adaptada às condições de estresse hídrico no seu crescimento e desenvolvimento inicial, como maior eficácia do sistema radicular (Vidal et al., 1999) e ajustamento osmótico, em função do acúmulo de carboidratos solúveis nas folhas e raízes (Chaves \& Stacciarini-Seraphin, 2001). Isso permite que ocupe e sobreviva em ambientes degradados e áreas descobertas (Oliveira-Filho \& Oliveira, 1988).

Schinus terebinthifolius apresenta alta plasticidade de colonização, estabelecendo-se em solos úmidos, arenosos, secos e arenosos a argilosos (Lenzi \& Orth, 2004). Devido a características como agressividade competitiva, tolerância higromórfica, boa interação biótica, caráter pioneiro, Schinus terebinthifolius apresenta forte potencial de regeneração em ambientes muito antropizados (Kageyama \& Gandara, 2000).

Das espécies utilizadas no plantio, apenas sete são exóticas, contudo uma delas, Leucaena leucocephala, é considerada espécie agressiva e inibidora da sucessão (Fonseca \& Jacobi, 2011). Esse fato é preocupante, pois essa espécie pode formar maciços adensados, excluir outras plantas do ecossistema e avançar rapidamente sobre áreas do entorno (Smith, 1985), principalmente 
em ambientes mais abertos. Portanto, é necessário a erradicação dos indivíduos dessa espécie exótica invasora e a substituição por mudas de espécies nativas.

A erradicação dos indivíduos de Leucaena leucocephala será facilitada pelo fato de, devido à curta idade do plantio (apenas 18 meses), ainda não ter ocorrido dispersão e germinação de sementes da espécie e regeneração na área, como observado no campo. Para as demais espécies exóticas plantadas, o monitoramento vai indicar a necessidade ou não de erradicação.

Cabe destacar que embora a utilização de espécies exóticas deva ser evitada, em determinadas situações, como no caso de solos expostos e sujeitos a erosão, tem sido recomendada por alguns autores para promover cobertura rápida e melhoria da fertilidade do solo, o que é particularmente importante no caso da mineração, além de possibilitar a formação de florestas catalisadoras, que facilitam a regeneração de espécies nativas no sub-bosque (Parrotta \& Knowles, 1999; Modna et al., 2010; Viani et al., 2010; Santilli \& Durigan, 2014). Nesse sentido, um estudo realizado por Santilli \& Durigan (2014) mostrou que mesmo numa comunidade em restauração em que $94 \%$ das árvores plantadas ( $86 \%$ das espécies) foram exóticas, após 8 anos do plantio apenas 3\% das plantas regenerantes pertenciam a espécies exóticas, indicando tendência de aumento da similaridade florística com um ecossistema natural de referência ao longo do tempo. Nesse estudo, espécies plantadas consideradas agressivas, como Leucaena leococephala e Acacia mangium, não apresentaram regenerantes após 8 anos do plantio (Santilli \& Durigan, 2014).

A presença de fragmentos florestais bem conservados no entorno da área em restauração avaliada neste estudo facilitará o enriquecimento natural, como destacam Martins (2014). Assim, espera-se uma tendência de substituição das poucas espécies exóticas plantadas por espécies nativas, mas de qualquer forma a erradicação de Leucaena leococephala já na fase inicial do reflorestamento é indicada como medida preventiva.

Considerando todos os indivíduos amostrados, as duas espécies com maior valor de importância (VI) foram Solanum lycocarpum (14,7\%) e Schinus terebinthifolius $(10,8 \%)$ (Figura 1). Com relação às famílias, destaque para Fabaceae e Solanaceae, perfazendo $41,0 \%$ do VI total.

Na distribuição das espécies por categoria sucessional, $28,9 \%$ das espécies plantadas pertencem ao grupo das pioneiras, $44,4 \%$ ao grupo das secundárias iniciais, $20 \%$ ao grupo das secundárias tardias e $6,7 \%$ não foram classificadas. Em nível de indivíduos, houve maior porcentagem da categoria secundária inicial, com $46,3 \%$ dos indivíduos, seguidos pelas da categoria pioneiras, com 39,1\% (Figura 2).

O plantio em maior densidade de espécies pioneiras e secundárias iniciais é bastante utilizado em reflorestamentos para restauração de áreas degradadas, pois essas espécies apresentam crescimento e desenvolvimento mais rápido e proporcionam condições edafoclimáticas favoráveis

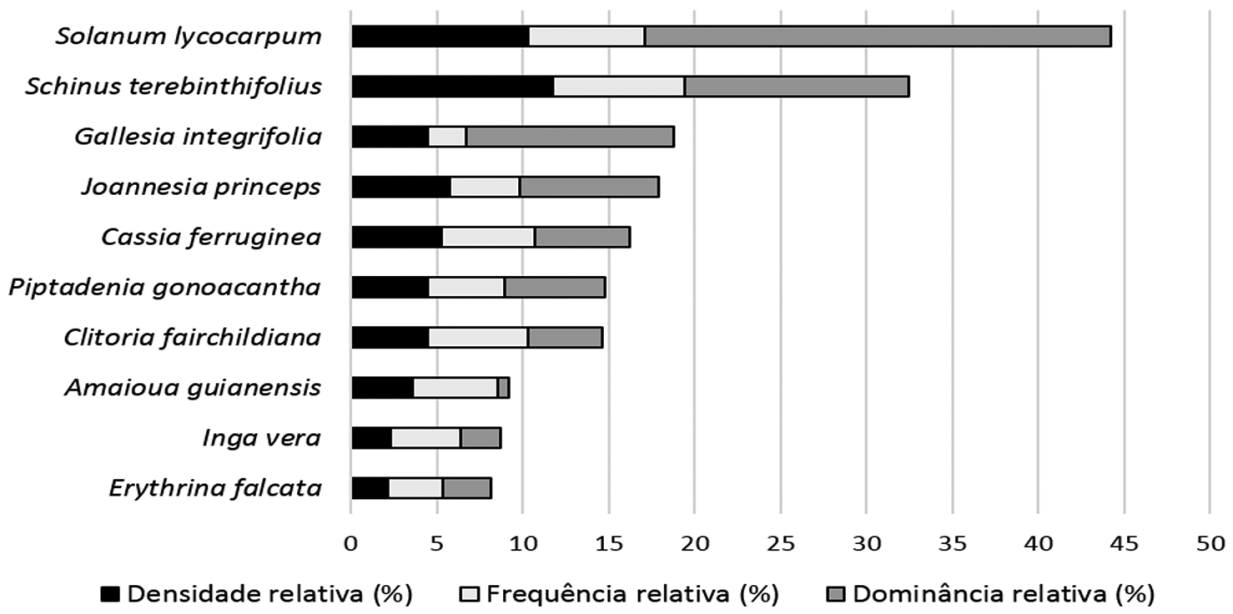

Figura 1. Espécies que apresentaram maior valor de importância (VI).

Figure 1. Species with highest importance values (VI). 


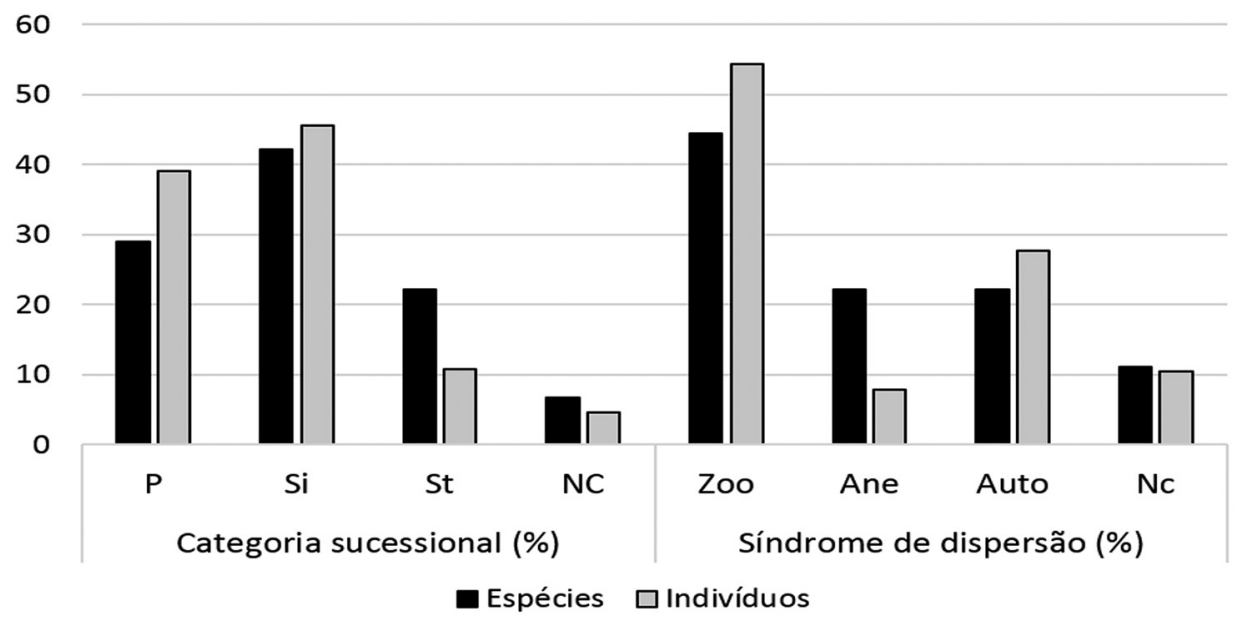

Figura 2. Distribuição por categoria sucessional e síndrome de dispersão das espécies e indivíduos pertencentes ao plantio da área em restauração, Descoberto, MG. P = pioneira; $\mathrm{Si}=$ secundária inicial; St = secundária tardia; $\mathrm{NC}=$ não classificada; Zoo = zoocoria; Ane = anemocoria; Auto $=$ autocoria $\mathrm{Nc}=$ não classificada.

Figure 2. Distribution by successional category and dispersion syndrome, of species and individuals belonging to planting, of the restoration site, Descoberto, MG. P = pioneer; SI = initial secondary; ST = late secondary; $\mathrm{NC}=$ unclassified; Zoo = zoochoric; Ane = anemocoric; Auto = autochory; Nc = unclassified.

ao desenvolvimento das espécies tardias (Ferreti \& Britez, 2005; Kageyama \& Gandara, 2005). Porém, uma densidade elevada de espécies pertencentes ao grupo das pioneiras pode comprometer os processos ecológicos futuros da área em restauração (Rodrigues et al., 2010), principalmente quando a área em restauração estiver em local distante de fontes de propágulos (fragmentos florestais) ou desprovidas de sementes armazenadas no solo, e, assim, inviabilizar o processo de sucessão florestal (Brancalion et al., 2009). Contudo, como nas proximidades da área deste estudo em restauração existem remanescentes florestais em estágio médio a avançado de regeneração, o enriquecimento com espécies finais de sucessão deverá ocorrer de forma natural.

A proporção de espécies e indivíduos com dispersão zoocórica se sobressaiu das demais, com 44,5\% de espécies e 54,3\% de indivíduos. A dispersão anemocórica foi a segunda maior em nível de espécies (Figura 2).

A utilização de espécies zoocóricas em plantios de restauração é importante para a atração da fauna dispersora. Animais dispersores de propágulos são fundamentais no favorecimento da complexidade de interações ecológicas e a relação planta-frugívoro se torna essencial na aceleração da sucessão florestal de áreas em restauração (Barbosa et al., 2012).

As espécies que mais contribuíram para a cobertura de copa foram Schinus terebinthifolius, Solanum lycocarpum e Joannesia princeps, perfazendo 60,4\% de cobertura total de copa (Tabela 2). Além da elevada densidade de mudas dessas espécies no plantio, a arquitetura dessas copas, que são tipicamente espécies de recobrimento ou preenchimento, contribuiu para o maior recobrimento proporcionado por essas espécies.

Em projetos de restauração realizados por meio de plantios baseados nos princípios da sucessão secundária é esperado que a cobertura de copas tenha a função de interferir no regime de luz e diminuir o impacto das gotas de chuvas no solo (Ignácio et al., 2007). Segundo os mesmos autores, isso pode promover, respectivamente, o sombreamento do terreno e a estabilização do solo. Desse modo, o rápido sombreamento tem a capacidade de diminuir ou até acabar com as ervas invasoras que competem com as mudas, além de fornecer condições microambientais favoráveis para o estabelecimento de espécies de sucessão mais avançada (Ignácio et al., 2007). Esse fato pode ser observado na área do presente estudo, na qual, em alguns locais em que a cobertura de copa já permitia um sombreamento mais denso sobre o solo, as gramíneas apresentavam sinais de senescência.

Com relação à altura média das plantas utilizadas no plantio, algumas espécies pioneiras e secundárias iniciais se sobressaíram em relação às demais espécies da mesma categoria sucessional, como Acacia mangium, 
Tabela 2. Estrutura das espécies pertencentes ao plantio da área em restauração.

Table 2. Structure of species belonging to planting of the restoration site.

\begin{tabular}{|c|c|c|c|c|c|c|c|c|}
\hline Espécies & $\mathrm{AC}\left(\mathrm{m}^{2}\right)$ & \%Copa & $\begin{array}{c}\text { Amin } \\
(\mathbf{m})\end{array}$ & $\begin{array}{c}A \max \\
(\mathrm{m})\end{array}$ & $\begin{array}{c}\text { Amed } \\
(\mathbf{m})\end{array}$ & $\begin{array}{c}\text { Dmin } \\
(\mathrm{cm})\end{array}$ & $\begin{array}{c}\text { Dmax } \\
(\mathrm{cm})\end{array}$ & $\begin{array}{c}\text { Dmed } \\
(\mathrm{cm})\end{array}$ \\
\hline Acacia mangium & 2,38 & 0,22 & 3,9 & 3,9 & 3,9 & 8,0 & 8,0 & 8,0 \\
\hline Amaioua guianensis & 21,49 & 1,99 & 0,7 & 2,3 & 1,3 & 0,6 & 4,5 & 2,1 \\
\hline Anadenanthera peregrina & 16,20 & 1,50 & 1,1 & 3,5 & 1,9 & 0,8 & 6,8 & 3,7 \\
\hline Annona muricata & 0,76 & 0,07 & 1,4 & 1,4 & 1,4 & 2,9 & 2,9 & 2,9 \\
\hline Bauhinia forficata & 8,53 & 0,79 & 0,8 & 2,6 & 1,6 & 1,3 & 5,4 & 3,1 \\
\hline Byrsonima sericea & 0,32 & 0,03 & 1,1 & 1,1 & 1,1 & 1,6 & 1,6 & 1,6 \\
\hline Caesalpinia peltophoroides & 5,40 & 0,50 & 1,6 & 2,0 & 1,8 & 3,5 & 5,7 & 4,8 \\
\hline Cariniana estrellensis & 4,64 & 0,43 & 1,2 & 2,3 & 1,8 & 2,2 & 4,5 & 3,2 \\
\hline Cassia ferruginea & 69,98 & 6,48 & 0,8 & 4,5 & 2,3 & 2,2 & 9,9 & 5,1 \\
\hline Cecropia hololeuca & 1,08 & 0,10 & 1,2 & 5,0 & 3,1 & 1,8 & 8,0 & 4,9 \\
\hline Cedrela fissilis & 0,65 & 0,06 & 0,3 & 0,6 & 0,4 & 0,6 & 4,8 & 2,7 \\
\hline Ceiba speciosa & 2,38 & 0,22 & 1,2 & 2,5 & 1,8 & 3,7 & 9,6 & 6,3 \\
\hline Clitoria fairchildiana & 37,37 & 3,46 & 0,6 & 4,0 & 2,2 & 1,1 & 10,8 & 4,7 \\
\hline Cordia magnoliifolia & 0,86 & 0,08 & 0,8 & 0,8 & 0,8 & 3,8 & 3,8 & 3,8 \\
\hline Cybistax antisyphilitica & 0,32 & 0,03 & 2,0 & 2,0 & 2,0 & 4,8 & 4,8 & 4,8 \\
\hline Enterolobium contortisiliquum & 0,22 & 0,02 & 1,6 & 1,6 & 1,6 & 3,2 & 3,2 & 3,2 \\
\hline Eriobotrya japonica & 2,59 & 0,24 & 1,2 & 1,6 & 1,5 & 2,0 & 3,6 & 2,8 \\
\hline Erythrina falcata & 5,51 & 0,51 & 1,3 & 2,7 & 1,8 & 3,2 & 8,9 & 5,8 \\
\hline Erythrina verna & 3,89 & 0,36 & 1,0 & 2,5 & 1,8 & 4,1 & 12,4 & 7,0 \\
\hline Gallesia integrifolia & 64,91 & 6,01 & 1,3 & 4,4 & 3,0 & 2,9 & 15,3 & 8,2 \\
\hline Genipa americana & 1,73 & 0,16 & 1,5 & 2,0 & 1,8 & 3,6 & 5,1 & 4,3 \\
\hline Genipa infudibuliformis & 4,75 & 0,44 & 0,6 & 1,8 & 1,1 & 1,3 & 2,6 & 2,1 \\
\hline Guarea macrophylla & 0,76 & 0,07 & 1,1 & 1,3 & 1,2 & 2,4 & 3,5 & 2,8 \\
\hline Hymenaea courbaril & 2,05 & 0,19 & 0,5 & 1,6 & 0,9 & 1,6 & 2,6 & 2,0 \\
\hline Inga vera & 22,61 & 2,09 & 1,2 & 3,0 & 1,8 & 1,6 & 10,2 & 4,9 \\
\hline Joannesia princeps & 74,83 & 6,93 & 1,0 & 5,0 & 2,5 & 1,9 & 12,7 & 5,9 \\
\hline Leucaena leucocephala & 9,99 & 0,93 & 0,9 & 4,0 & 2,3 & 1,9 & 5,1 & 3,4 \\
\hline Maprounea guianensis & 0,05 & 0,00 & 1,5 & 1,5 & 1,5 & 3,4 & 3,4 & 3,4 \\
\hline Myroxylon peruiferum & 10,56 & 0,98 & 1,7 & 5,0 & 3,2 & 3,1 & 8,3 & 5,2 \\
\hline Pachira glabra & 5,13 & 0,47 & 1,1 & 2,1 & 1,6 & 1,9 & 4,8 & 3,3 \\
\hline Peltophorum dubium & 7,16 & 0,66 & 2,5 & 4,1 & 3,1 & 4,5 & 7,0 & 6,2 \\
\hline Piptadenia gonoacantha & 69,45 & 6,43 & 1,2 & 4,5 & 2,9 & 1,0 & 11,1 & 5,6 \\
\hline Plathymenia reticulata & 7,06 & 0,65 & 1,9 & 2,5 & 2,1 & 3,8 & 8,3 & 7,3 \\
\hline Sapindus saponaria & 0,79 & 0,07 & 1,9 & 1,9 & 1,9 & 3,0 & 3,0 & 3,0 \\
\hline Schinus terebinthifolius & 293,47 & 27,17 & 0,8 & 4,5 & 2,1 & 2,9 & 12,1 & 5,3 \\
\hline Schizolobium parahyba & 3,14 & 0,29 & 1,8 & 1,8 & 1,8 & 4,1 & 4,1 & 4,1 \\
\hline Seguieria langsdorffii & 3,06 & 0,28 & 1,3 & 3,3 & 2,6 & 4,0 & 11,5 & 8,0 \\
\hline Senna macranthera & 0,79 & 0,07 & 2,1 & 2,1 & 2,1 & 2,9 & 2,9 & 2,9 \\
\hline Senna multijuga & 0,79 & 0,07 & 2,4 & 2,4 & 2,4 & 1,9 & 1,9 & 1,9 \\
\hline Solanum lycocarpum & 283,62 & 26,26 & 1,2 & 5,0 & 2,9 & 1,8 & 20,1 & 7,6 \\
\hline Solanum mauritianum & 0,95 & 0,09 & 3,5 & 3,5 & 3,5 & 4,8 & 4,8 & 4,8 \\
\hline Solanum paniculatum & 9,15 & 0,85 & 2,1 & 4,4 & 3,5 & 9,9 & 21,0 & 14,2 \\
\hline Syzygium cumini & 7,95 & 0,74 & 0,9 & 1,8 & 1,4 & 1,0 & 4,8 & 2,6 \\
\hline Terminalia catappa & 8,34 & 0,77 & 0,7 & 3,1 & 1,8 & 1,0 & 9,9 & 3,5 \\
\hline Zeyheria tuberculosa & 2,36 & 0,22 & 1,8 & 2,5 & 2,2 & 2,8 & 4,9 & 4,0 \\
\hline Total & $1.080,00$ & 100,00 & & & & & & \\
\hline
\end{tabular}

$\mathrm{AC}=$ área de copa $;$ Amin = altura mínima; Amax = altura máxima; Amed = altura média; Dmin = diâmetro mínimo; Dmax = diâmetro máximo; Dmed = diâmetro médio. 
Cecropia hololeuca, Solanum mauritianum, Solanum paniculatum e Peltophorum dubium (Tabela 2).

Algumas espécies secundárias tardias se sobressaíram em relação à algumas espécies de início de sucessão (pioneiras e secundárias iniciais), como Myroxylon peruiferum, Sapindus saponaria, Genipa americana, Cariniana estrellensis e Ceiba speciosa (Tabelas 1 e 2). Um dos fatores que pode ter contribuído para esse fato foi a grande densidade do Cajanus cajan que, ao proporcionar um maior sombreamento, provavelmente favoreceu melhor desenvolvimento das espécies tardias perante algumas pioneiras e secundárias iniciais. Esse fato contribui de forma positiva para o processo de restauração da área, pois são as espécies finais de sucessão que irão garantir a perpetuação da área plantada (Rodrigues et al., 2009).

A altura média das mudas plantadas variou de 0,4 $\mathrm{m}$ a 3,9 $\mathrm{m}$. Quanto ao diâmetro médio das mudas, destacaram-se as pioneiras Solanum paniculatum, Acacia mangium e Solanum lycocarpum e as secundárias iniciais Gallesia integrifolia e Seguieria langsdorffi, que obtiveram os maiores diâmetros (Tabela 2). Entre as espécies secundárias tardias se destacaram Ceiba speciosa, Myroxylon peruiferum e Genipa americana, que obtiveram diâmetro médio superior ao das outras 22 espécies, entre essas diversas pioneiras e secundárias iniciais.

As espécies que se destacaram tanto em altura média quanto em área basal (diâmetro médio) e cobertura de copa se mostraram potencialmente favoráveis para a restauração de áreas degradadas por mineração de bauxita, pois proporcionaram mais rápida cobertura do solo. Esse fato é desejável em áreas em restauração, para que a sucessão avance rapidamente, propicie o desenvolvimento do estrato de regeneração natural e forme uma barreira ao desenvolvimento de gramíneas invasoras, além de protegerem o solo recomposto de processos erosivos. As espécies com maior destaque, ao analisar os três parâmetros conjuntamente, foram: Anadenanthera peregrina, Solanum paniculatum, Solanum lycocarpum, Myroxylon peruiferum, Gallesia integrifolia e Schinus terebinthifolius.

A restauração através do plantio de mudas tem a capacidade de mitigar muitos efeitos e impactos ambientais, permitindo que a área consiga restabelecer algumas de suas características existentes antes do impacto ou degradação sofrida, como a intensificação das interações ecossistêmicas e a recomposição da camada superficial de solo (Pereira et al., 2012). Mas, segundo esses autores, é importante o conhecimento da ecologia das espécies que serão utilizadas, pois cada uma possui ritmo de crescimento e desenvolvimento diferenciado e esse conhecimento é primordial para o sucesso de um projeto de restauração florestal.

\section{CONCLUSÕES}

As espécies empregadas no plantio para a restauração da área em estudo, mesmo no seu estágio inicial de desenvolvimento, já proporcionam benefícios ecológicos como cobertura do solo através das copas, aspecto fundamental para evitar processos erosivos e invasão de gramíneas exóticas agressivas.

\section{AGRADECIMENTOS}

$\mathrm{O}$ autores agradecem à Votorantim Metais pelo financiamento do projeto, ao $\mathrm{CNPq}$ pelas Bolsas de Mestrado e atualmente Doutorado da primeira autora e de Produtividade em Pesquisa do segundo autor, e à CAPES pela Bolsa de Doutorado do terceiro autor

\section{STATUS DA SUBMISSÃO}

Recebido: 15 abr., 2015

Aceito: 28 nov., 2015

\section{*AUTOR(ES) PARA CORRESPONDÊNCIA}

\section{Kelly de Almeida Silva}

Departamento de Engenharia Florestal, Universidade Federal de Viçosa - UFV, Avenida Peter Henry Rolfs, s/n, Campus Universitário, CEP 36570-900, Viçosa, MG, Brasil e-mail: kellyalmeidaenf@yahoo.com.br

\section{REFERENNCIAS}

Almeida ROPO, Sánchez LE. Revegetação de áreas de mineração: critérios de monitoramento e avaliação do desempenho. Revista Árvore 2005; 29(1): 47-54. http:// dx.doi.org/10.1590/S0100-67622005000100006.

Angiosperm Phylogeny Group III - APG. An update of the Angiosperm Phylogeny Group classification for the orders and families of flowering plants. Botanical Journal 
of the Linnean Society 2009; 161(2): 105-121. http://dx.doi. org/10.1111/j.1095-8339.2009.00996.x.

Azevedo RL, Ribeiro GT, Azevedo CLL. Feijão guandu: uma planta multiuso. Revista da Fapese 2007; 3(2): 81-86.

Barbosa JM, Eisenlohr PV, Rodrigues MA, Barbosa KV. Ecologia da dispersão de sementes em florestas tropicais. In: Martins SV, editor. Ecologia de florestas tropicais do Brasil. Viçosa: Editora UFV; 2012.

Beltrame TP, Rodrigues E. Comparação de diferentes densidades de feijão guandu (Cajanus cajan (L.) Millsp.) na restauração florestal de uma área de reserva legal no Pontal do Paranapanema, SP. Scientia Forestalis 2008; 36(80): 317-327.

Brancalion PHS, Isernhagen I, Gandolfi S, Rodrigues RR. Fase 2: plantio de árvores nativas brasileiras fundamentada na sucessão florestal. In: Rodrigues RR, Brancalion PHS, Isernhagen I, editores. Pacto pela restauração da Mata Atlântica: referencial dos conceitos e ações de restauração florestal. São Paulo: Instituto BioAtlântica; 2009.

Brancalion PHS, Viani RAG, Rodrigues RR, Gandolfi S. Avaliação e monitoramento de áreas em processo de restauração. In: Martins SV, editor. Restauração ecológica de ecossistemas degradados. 2. ed. Viçosa: Editora UFV; 2015.

Chaves JT Fo, Stacciarini-Seraphin E. Alteração no potencial osmótico e teor de carboidratos solúveis em plantas jovens de lobeira (Solanum lycocarpum St.-Hil.) em resposta ao estresse hídrico. Brazilian Journal of Botany 2001; 24(2): 199-204.

Chazdon RL. Beyond deforestation: restoring forests and ecosystem services on degraded lands. Science 2008; 320(5882): 1458-1460. http://dx.doi.org/10.1126/ science.1155365. PMid:18556551.

Courtney R, Mullen G, Harrington T. An Evaluation of Revegetation Success on Bauxite Residue. Restoration Ecology 2009; 17(3): 350-358. http://dx.doi.org/10.1111/j.1526100X.2008.00375.x.

Ferreti AR, Britez RM. A restauração da Floresta Atlântica no litoral do Estado do Paraná: os trabalhos da SPVS. In: Galvão APM, Porfírio-da-Silva V, editores. Restauração florestal: fundamentos e estudos de caso. Colombo: EMBRAPA; 2005.

Fonseca NG, Jacobi CM. Desempenho germinativo da invasora Leucaena leucocephala (Lam.) de Wit. e comparação com Caesalpinia ferrea Mart. ex Tul. e Caesalpinia pulcherrima (L.) Sw. (Fabaceae). Acta Botanica Brasílica 2011; 25(1): 191-197. http://dx.doi.org/10.1590/ S0102-33062011000100022.

Gandolfi S, Leitão Filho HF, Bezerra CLF. Levantamento florístico e caráter sucessional das espécies arbustivoarbóreas de uma floresta mesófila semidecídua no município de Guarulhos, SP. Revista Brasileira de Biologia 1995; 55(4): 753-767.
Grant CD, Ward SC, Morley SC. Return of ecosystem function to restored bauxite mines in western Australia. Restoration Ecology 2007;15(4, Supl): S94-S103. http:// dx.doi.org/10.1111/j.1526-100X.2007.00297.x.

Ignácio ED, Attanasio CM, Toniato MTZ. Monitoramento de plantios de restauração de florestas ciliares: microbacia do Ribeirão São João, Mineiros do Tietê, SP. Revista Instituto Florestal 2007; 19(2): 137-148.

Instituto Brasileiro de Geografia e Estatística - IBGE. Manual técnico da vegetação brasileira. 2 . ed. Rio de Janeiro; 2012. Manuais Técnicos em Geociências.

Jardim Botânico do Rio de Janeiro - JBRJ. Reflora. Lista de Espécies da Flora do Brasil. Rio de Janeiro; 2014 [citado em 2015 abril 15]. Disponível em:http://floradobrasil. jbrj.gov.br/

Jefferson LV. Implications of plant density on the resulting community structure of mine site land. Restoration Ecology 2004; 12(3): 429-438. http://dx.doi.org/10.1111/j.10612971.2004.00328.x.

Kageyama P, Gandara FB. Resultados do programa de restauração com espécies arbóreas nativas do convênio Esalq/USP e CESP. In: Galvão APM, Porfírio-da-Silva V, editores. Restauração florestal: fundamentos e estudos de caso. Colombo: EMBRAPA; 2005.

Kageyama P, Gandara FB. Revegetação de áreas ciliares. In: Rodrigues RR, Leitão HF Fo, editores. Matas ciliares: conservação e recuperação. São Paulo: EDUSP; 2000.

Leite LFC, Freitas RCA, Sagrilo E, Galvão SRS. Decomposição e liberação de nutrientes de resíduos vegetais depositados sobre Latossolo Amarelo no Cerrado Maranhense. Revista Ciência Agronômica 2010; 41(1): 29-35.

Lenzi M, Orth AF. Fenologia reprodutiva, morfologia e biologia floral de Schinus terebinthifolius Raddi (Anacardiaceae), em restinga da Ilha de Santa Catarina, Brasil. Biotemas 2004; 17(2): 67-89.

Lopes RF, Branquinho JA. Jazidas de bauxita da Zona da Mata de Minas Gerais. In: Schobbenhaus C, Coelho CES, coordenadores. Principais depósitos minerais do Brasil. Brasília: Departamento Nacional da Produção Mineral; 1988. v. 3.

Martins SV, Kunz S. Use of evaluation and monitoring indicators in a riparian forest restoration project in Viçosa, Southeastern Brazil. In: Rodrigues RR, Martins SV, Gandolfi S, editores. High diversity forest restoration in degraded areas. New York: Nova Science Publishers; 2007.

Martins SV. Recuperação de matas ciliares. 3. ed. Viçosa: Aprenda Fácil; 2014.

Miranda A No, Martins SV, Silva KA, Gleriani JM. Florística e estrutura do estrato arbustivo-arbóreo de uma floresta restaurada com 40 anos, Viçosa, MG. Revista Árvore 2012; 36(5): 869-878. http://dx.doi.org/10.1590/ S0100-67622012000500009. 
Modna D, Durigan G, Vital MVC. Pinus elliottii Engelm como facilitadora da regeneração natural da mata ciliar em região de Cerrado, Assis, SP, Brasil. Scientia Forestalis 2010; 38(85): 73-83.

Moro MF, Martins FR. Métodos de levantamento do componente arbóreo-arbustivo. In: Felfili JM, Eisenlohr PV, Melo MMRF, Andrade LA, Meira JAA No, editores. Fitossociologia no Brasil: métodos e estudos de casos. Viçosa: Editora UFV; 2011.

Mueller-Dombois D, Ellenberg H. Aims and methods of vegetation ecology. New York: John Wiley \& Sons; 1974.

Oliveira-Filho AT, Oliveira LCA. Biologia floral de uma população de Solanum lycocarpum A. St. Hil. (Solanaceae) em Lavras, MG. Revista Brasileira de Botanica. Brazilian Journal of Botany 1988; 11(1/2): 23-32.

Oliveira-Júnior EN, Santos CD, Abreu CMP, Corrêa AD, Santos JZL. Análise nutricional da fruta-de-lobo (Solanum lycocarpum St. Hil.) durante o amadurecimento. Ciência e Agrotecnologia 2003; 27(4): 846-851. http://dx.doi. org/10.1590/S1413-70542003000400016.

Parrotta JA, Knowles OH. Restoration of tropical moist forests on bauxite-mined lands in Brazilian Amazon. Restoration Ecology 1999; 7(2): 103-116. http://dx.doi. org/10.1046/j.1526-100X.1999.72001.x.

Pereira JS, Abreu CFNR, Pereira RA Jr, Rodrigues SC. Avaliação do índice de sobrevivência e crescimento de espécies arbóreas utilizadas na recuperação de área degradada. Revista Geonorte 2012; 1(4): 138-148.

Pijl L van der. Principles of dispersal in higher plants. 3. ed. Berlin: Springer-Verlag; 1982.

Rodrigues ER, Monteiro R, Cullen L Jr. Dinâmica inicial da composição florística de uma área restaurada na região do Pontal do Paranapanema, São Paulo, Brasil. Revista Árvore 2010; 34(5): 853-861. http://dx.doi.org/10.1590/ S0100-67622010000500010.
Rodrigues RR, Lima RAF, Gandolfi S, Nave AG. On the restoration of high diversity forests: 30 years of experience in the Brazilian Atlantic Forest. Biological Conservation 2009; 142(6): 1242-1251. http://dx.doi.org/10.1016/j. biocon.2008.12.008.

Sá A Jr. Aplicação da classificação de Köppen para o zoneamento climático do Estado de Minas Gerais [dissertação]. Lavras: Universidade Federal de Lavras; 2009.

Santilli C, Durigan G. Do alien species dominate plant communities undergoing restoration? A case study in the Brazilian savanna. Scientia Forestalis 2014; 42(103): 371-382.

Schievenin DF, Tonello KC, Silva DA, Valente ROA, Faria LC, Thiersch CR. Monitoramento de indicadores de uma área de restauração florestal em Sorocaba - SP. Revista Científica Eletrônica de Engenharia Florestal 2012; 19(1): 95-108.

Shepherd GJ. Fitopac 2.1. Campinas: Departamento de Botânica, Universidade Estadual de Campinas; 2010.

Smith CW. Impact of alien plants on Hawaii's native biota. In: Stone CPJ, Scott M, editores. Hawaii's terrestrial ecosystems: preservation and management. Manoa: Cooperative National Park Resources Studies Unit, University of Hawaii; 1985.

U.S. Geological Survey - USGS. Mineral Commodity Summaries 2015. Virginia; 2015.

Viani RAG, Durigan G, Melo ACG. A regeneração natural sob plantações florestais: desertos verdes ou redutos de biodiversidade? Ciência Florestal 2010; 20(3): 533-552. http://dx.doi.org/10.5902/198050982067.

Vidal MC, Stacciarini-Seraphin E, Câmara HHLL. Crescimento de plântulas de Solanum lycocarpum St. Hil. (lobeira) em casa de vegetação. Acta Botanica Brasílica 1999; 13(3): 271-275. 